\title{
Transforming in-situ observations of CME-driven shock accelerated protons into the shock's reference frame.
}

\author{
I. M. Robinson and G. M. Simnett \\ Physics and Astronomy, University of Birmingham, UK \\ Received: 1 December 2004 - Revised: 23 March 2005 - Accepted: 14 April 2005 - Published: 28 July 2005
}

\begin{abstract}
We examine the solar energetic particle event following solar activity from 14, 15 April 2001 which includes a "bump-on-the-tail" in the proton energy spectra at $0.99 \mathrm{AU}$ from the Sun. We find this population was generated by a CME-driven shock which arrived at $0.99 \mathrm{AU}$ around midnight 18 April. As such this population represents an excellent opportunity to study in isolation, the effects of proton acceleration by the shock. The peak energy of the bump-onthe-tail evolves to progressively lower energies as the shock approaches the observing spacecraft at the inner Lagrange point. Focusing on the evolution of this peak energy we demonstrate a technique which transforms these in-situ spectral observations into a frame of reference co-moving with the shock whilst making allowance for the effects of pitch angle scattering and focusing. The results of this transform suggest the bump-on-the-tail population was not driven by the 15 April activity but was generated or at least modulated by a CME-driven shock which left the Sun on 14 April. The existence of a bump-on-the-tail population is predicted by models in Rice et al. (2003) and Li et al. (2003) which we compare with observations and the results of our analysis in the context of both the 14 April and 15 April CMEs. We find an origin of the bump-on-the-tail at the 14 April CME-driven shock provides better agreement with these modelled predictions although some discrepancy exists as to the shock's ability to accelerate $100 \mathrm{MeV}$ protons.
\end{abstract}

Keywords. Solar physics, astrophysics and astronomy (Energetic particles; Flares and mass ejections) - Space plasma physics (Transport processes)

\section{Introduction}

Interplanetary energetic particles represent a valuable tool for the remote sensing of solar activity. The Sun drives the majority of energetic particle events seen at L1, either through solar flares or the release of coronal mass ejections

Correspondence to: I. Robinson

(imr@star.sr.bham.ac.uk)
(CMEs). Both phenomena are known to accelerate charged particles although the details of the acceleration processes remain a matter of current research. Whilst the energetic particles themselves may present signatures of the acceleration processes they are often superimposed in the interplanetary medium given the generally small overlap $( \pm 10$ $15 \mathrm{~min}$ ) between flare and CME onset. When combined with the uncertainties of interplanetary propagation, such as the configuration of the interplanetary magnetic field and the level of pitch angle scattering, some doubt can exist as to the precise origin of solar energetic particle events. Additional diagnostics are available if one investigates particle ionisation states and abundance ratios in the interplanetary medium. These have been shown to distinguish between flare and CME particle populations (see Reames, 1999, for a review) although doubts have been raised (Klein and Trottet, 2001) as to the validity of such approaches at energies above $\sim 10 \mathrm{MeV} /$ nucleon. The active region is probably incapable of supplying all the energy for a large flare and it has been suggested (Simnett, 2003) that the coronal seed population provides a significant part of the flare energy, and that the active region at the time of the flare produces the high end of the proton spectrum i.e. the GeV particles. Then the total output from the flare is available to the CME, which may provide further acceleration and re-distribution (Torsti et al., 2002; Tylka et al., 2002).

Here we attempt to clarify our view of global acceleration by isolating a characteristic proton population released from a CME-driven shock which is manifest as a bump-on-thetail of the in-situ proton spectra upstream of the shock. In so doing, we demonstrate a transformation of these observations into a frame of reference which was co-moving with the shock to find the most likely proton energy to escape the shock region as a function of the shock's position. We effectively use in-situ observations of the proton spectrum to map changes in the energetic proton output from the shock, to its position. We are motivated to do so in the hope that such analysis should provide an improved basis for the testing of models for particle acceleration at MHD-shocks. In particular we find our observations and analysis are in good 
qualitative agreement with the Rice et al. (2003) model for particle acceleration at CME-driven shocks which predicts the presence of a bump-on-the-tail feature in the proton spectra upstream of the shock. Regardless of this prediction we find, on an observational basis, that the only viable origin for this spectral feature (which we hereafter refer to as "the dynamic spectral peak" or "DSP"), is the shock itself as discussed in Sect. 4.

We focus on the 15-18 April 2001 solar energetic particle event which was the largest ground level event (GLE) of the current solar cycle, reaching $\mathrm{GeV}$ proton energies during its early stages. Neutron monitor studies of this solar energetic particle event (Bieber et al., 2004) suggest that the time of arrival of relativistic protons is consistent with an origin at a CME which erupted from the Sun's west limb at $\sim 13: 45$ UT 15 April. However, the timing uncertainties in their analysis do not preclude acceleration in the flare site. Indeed there is some reason to believe that high proton energies reached during this event may have been enhanced by some combination of flare and CME particle acceleration mechanism (Tylka et al., 2002). Regardless of this, some aspect of the 15 April solar activity seems the likely driver of the 15-18 April particle event. In contrast, the CME-transform employed here suggests the DSP was generated by a CME-driven shock which left the Sun on 14 April which in turn implies the particle event was either generated at least in part by this shock or, this shock "filtered" the output from the 15 April activity. Using speed, distance and time relations for protons contributing to the DSP, we calculate the shock's position and the time at which these protons were released, having taken into account changes in the net proton speed due to scattering. Starting with nominal proton velocities derived from the protons' energy, we then use the shock's velocity to correct these nominal velocities to allow for propagation delays. Consequently the analysis presented here could also provide some insight into the effects of pitch angle scattering in the interplanetary medium.

\section{Instrumentation}

To illustrate the frame of reference transformation we concentrate on the $0.06-4.75 \mathrm{MeV}$ proton intensities recorded by the Electron, Proton and Alpha Monitor or EPAM (Gold et al., 1998) following significant flare and CME activity on 15 April 2001. The EPAM detectors we are using cannot distinguish between the various ion species. However, for typical solar energetic particle events, the bulk of the detector response is from protons and we therefore refer to them as such here. EPAM is one of a suite of instruments carried by the Advanced Composition Explorer (ACE) which occupies a "halo" orbit about the inner Lagrange point (L1). The DSP spectral feature upon which we focus our attention here is best observed using EPAM's Multi-Frequency Spectral Analyser (MFSA) which produces a high resolution, 32 point energy spectrum with a $20 \mathrm{~min}$ cadance. Higher time resolution measurements are available with an 8 point energy spectrum, however, during the period we discuss there are no significant short term variations and we sacrifice this higher time resolution in favour of the best available proton energy resolution.

At the early stages of the 15 April event, all of EPAM's proton observations were subject to a contamination of the counting statistics where the apparent level of contamination became less significant at successively higher proton energy channels. The prompt rise in the lowest energy proton channels observed with EPAM ( $>61 \mathrm{keV} /$ nucleon), provides the evidence for this contamination since protons at these energies could not have arrived at EPAM so quickly following the observed solar activity. During the early stages of this event, ACE was bathed in a flux of high energy particles and the observed electron spectrum was exceptionally hard with a power-law spectral index of -1.3 . Consequently it is suspected that this contamination arises from $>1 \mathrm{MeV}$ electrons incident upon EPAM's solid state detectors where they may deposit varying fractions of their energy which is subsequently mis-interpreted in EPAM's measurements. A more detailed discussion of the cause of this contamination is beyond the scope of this paper. For our purposes we can minimise its effects by subtracting a characteristic powerlaw from the proton energy spectrum; this power-law is determined by fitting the proton spectrum at low energies i.e. those energies which are dominated by this contamination. This is sufficient to remove the bulk of the contamination whilst enhancing the spectral peak during the early stages of the event; at later stages the effects of this contamination rapidly diminish and are certainly insignificant before the end of April 16.

\section{Summary of the 15-18 April event}

The period of interest is shown in Fig. 1 with the proton intensities recorded by EPAM displayed in Fig. 1a covering a total energy range of 61-4750 keV/nucleon; the central energy of each proton channel is given by the colour-coded key. The intensity of $103-175 \mathrm{keV}$ electrons is also shown as the black line in this plot.

The initial rise in proton intensity at EPAM occurs at 14:17 UT 15 April 2001 and the effects of contamination in the lower energy proton channels can be easily identified in Fig. 1a by comparison with the $103-175 \mathrm{keV}$ electron intensities. These protons could not have arrived at EPAM so promptly and the different proton energy channels do not show any indication of velocity dispersion. Consequently we must conclude these channels have been subject to some form of contamination from relativistic particles, mainly electrons.

The "shock-spike" in the proton intensities corresponding to the arrival of the CME-driven shock at ACE can clearly be seen around midnight on 18 April. The solar wind speed data (Fig. 1b) recorded by the Solar Wind Electron, Proton and Alpha Monitor or SWEPAM (McComas et al., 1998) shows a discontinuous rise in solar wind speed to $\sim 500 \mathrm{~km} / \mathrm{s}$ whilst a 


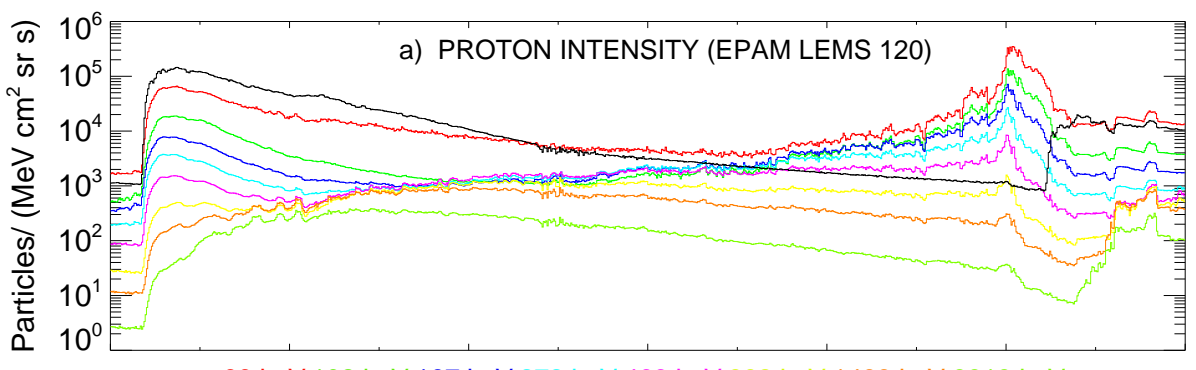

69 keV 102 keV 167 keV 272 keV 469 keV 862 keV 1499 keV 3313 keV
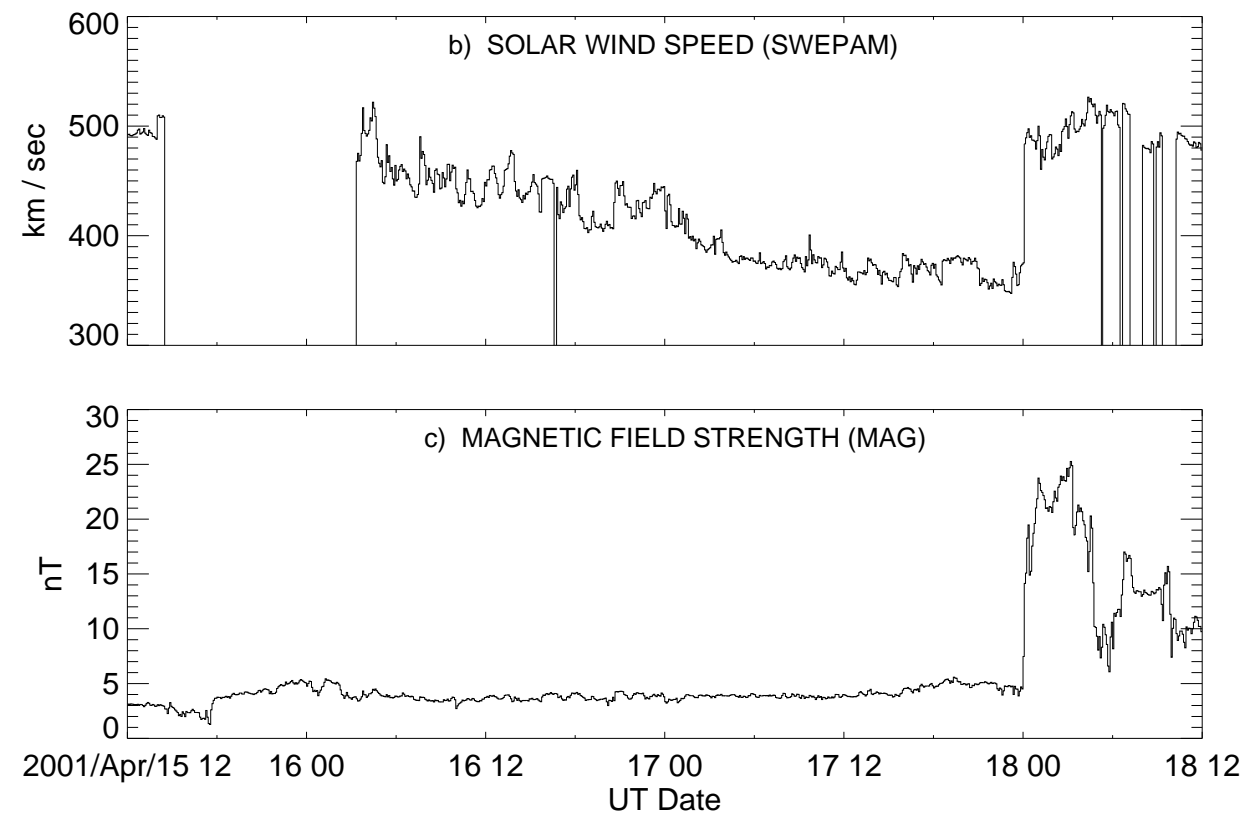

Fig. 1. Summary of observations at ACE for the 15-18 April event showing the proton intensities and the $103-175 \mathrm{keV}$ electron intensity observed by EPAM (a), the solar wind speed observed by SWEPAM (b) and the variation in the magnetic field strength recorded by MAG (c).

discontinuous rise in the magnetic field (Fig. 1c) observed by MAG (Smith et al., 1998) completes an almost unambiguous identification of the shock arrival at 0.99 AU from the Sun at 00:04 UT 18 April 2001.

Observations from the Large Angle Spectroscopic Coronagraph or LASCO (Brueckner et al., 1995) reveal the 15-18 April was briefly preceded by the eruption of a CME centred about the SW limb of the Sun with a projected plane-ofsky velocity of $\sim 1200 \mathrm{~km} / \mathrm{s}$; the projected lift-off time was $\sim 13: 33$ UT 15 April. Also an X14 X-ray flare was observed to peak at 13:50 UT by the Geostationary Operational Environment Satellites (GOES) originating from active region 9415 , at that time located $85 \mathrm{deg}$ west of the central meridian and $20 \mathrm{deg}$ south of the solar equator. Given the location of the flare on the very limb of the Sun, it is unlikely the bulk of the ejecta was directed along the Sun-Earth line. Observations of the solar wind environment at ACE clearly show the arrival of a CME-driven shock at $\mathrm{L} 1$ and it is possible we may have observed the extreme flank of this CME. In addition there may have been a contemporary ejection closer to the Sun-Earth line which was either unobserved by LASCO, or confused with the limb ejection. The relatively low shock speed we discuss in Sect. 5 supports the latter hypothesis of a contemporary ejection. An alternative scenerio where an earlier CME may have contributed to this event is discussed in Sect. 7 and may have been more likely to reach L1 on midnight 18 April. We find the most likely candidate, associated with an M1.0 X-ray flare $70 \mathrm{deg}$ west of the central meridian, had a plane of sky velocity of $\sim 750 \mathrm{~km} / \mathrm{s}$. This also seems more consistent with the in-situ shock speed measured at ACE, its presence near the Sun is observed rather than implied and does not require such a large angular width to have reached L1 on 18 April. For comparison, extrapolated height-time profiles for both the 14 April and 15 April CMEs are shown in Fig. 3.

By examining the evolution of the proton energy spectrum recorded by EPAM we can identify a bump-on-the-tail population or DSP in connection with this activity which we will show, can only be the result of acceleration at a CMEdriven shock and not the X14 flare. The application of the CME-transform then provides further insight suggesting the 14 April CME-driven shock was the more likely source of this spectral feature. 

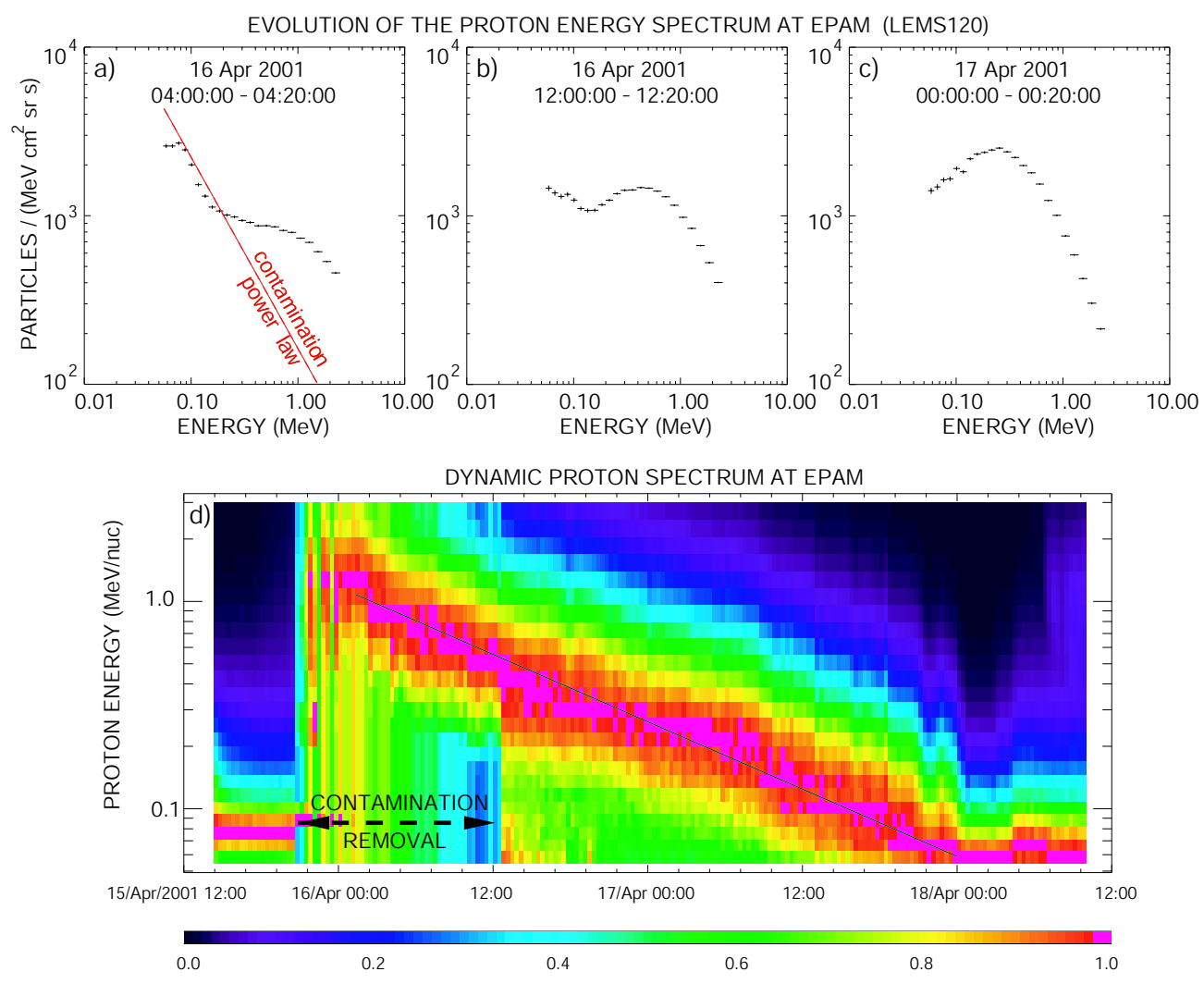

Fig. 2. Panels (a), (b) and (c) show the $20 \mathrm{~min}$ averaged, proton energy spectrum at various times throughout the $15-18$ April event. The bump-on-the-tail population can clearly be observed to rise above the pre-existing foreground (some of which is due to electron contamination of the proton intensity channels). The dynamic proton spectrum is shown in panel (d) and provides a convenient means to track the evolution of this proton population. We have removed the bulk of the effects of contamination from panel (d) (for the indicated period) by fitting the contaminated region of the spectrum (dashed red line in panel (a)), extrapolating and subtracting from the proton energy spectrum.

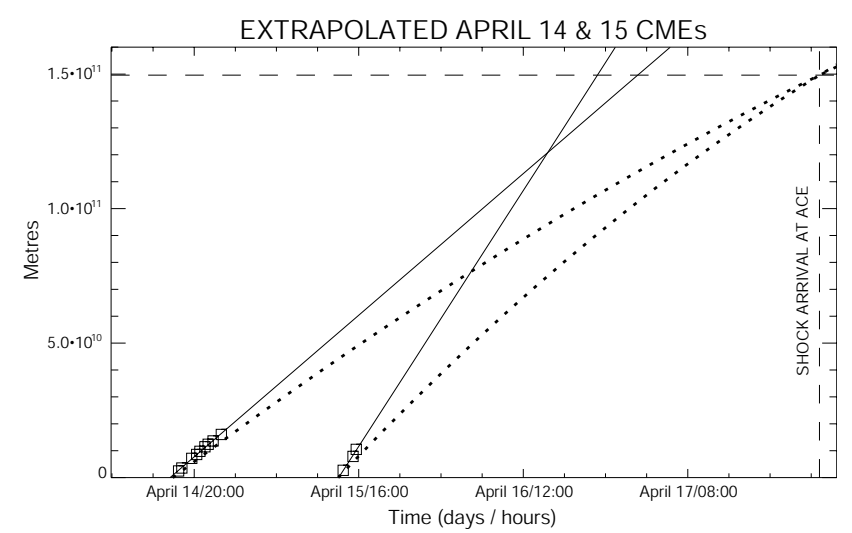

Fig. 3. Height-time plots based on LASCO observations of the 17:23 UT April 14 and 13:33 UT 15 April CMEs. The solid lines are a linear extrapolation of the LASCO observations out to $1 \mathrm{AU}$ whilst the dotted lines show a second order polynomial fit to the LASCO observation and the in-situ shock speed observed at ACE.

\section{The proton dynamic spectrum}

Figures $2 \mathrm{a}, \mathrm{b}$ and $\mathrm{c}$ show the evolution in the proton energy spectrum as the 15-18 April 2001 event progresses. The emergence and evolution of a spectral peak population can clearly be seen. This feature can be more easily tracked by viewing the dynamic proton spectrum shown in Fig. $2 d$ which uses a colour-scale to show the evolution of the $20 \mathrm{~min}$ averaged MFSA proton energy spectrum.

In this case the proton energy spectrum for each time interval is normalised to the peak proton intensity for that time interval to provide a clearer view of the change in the peak energy of the bump-on-the-tail population. The fact that the peak in the proton energy spectrum peaks at the lowest energy channel as the shock arrives is entirely coincidental, for example, this effect is not replicated either in the helium dynamic spectrum during this event, nor during every other DSP event we have studied. We have attempted to remove the contamination seen at low proton energies by fitting a power-law to the low energy portion of the proton energy spectrum (dashed red line in Fig. 2a), extrapolating and subtracting from the spectrum. This was only necessary at the early stages of the event as indicated by the dashed black line in Fig. 2d. 

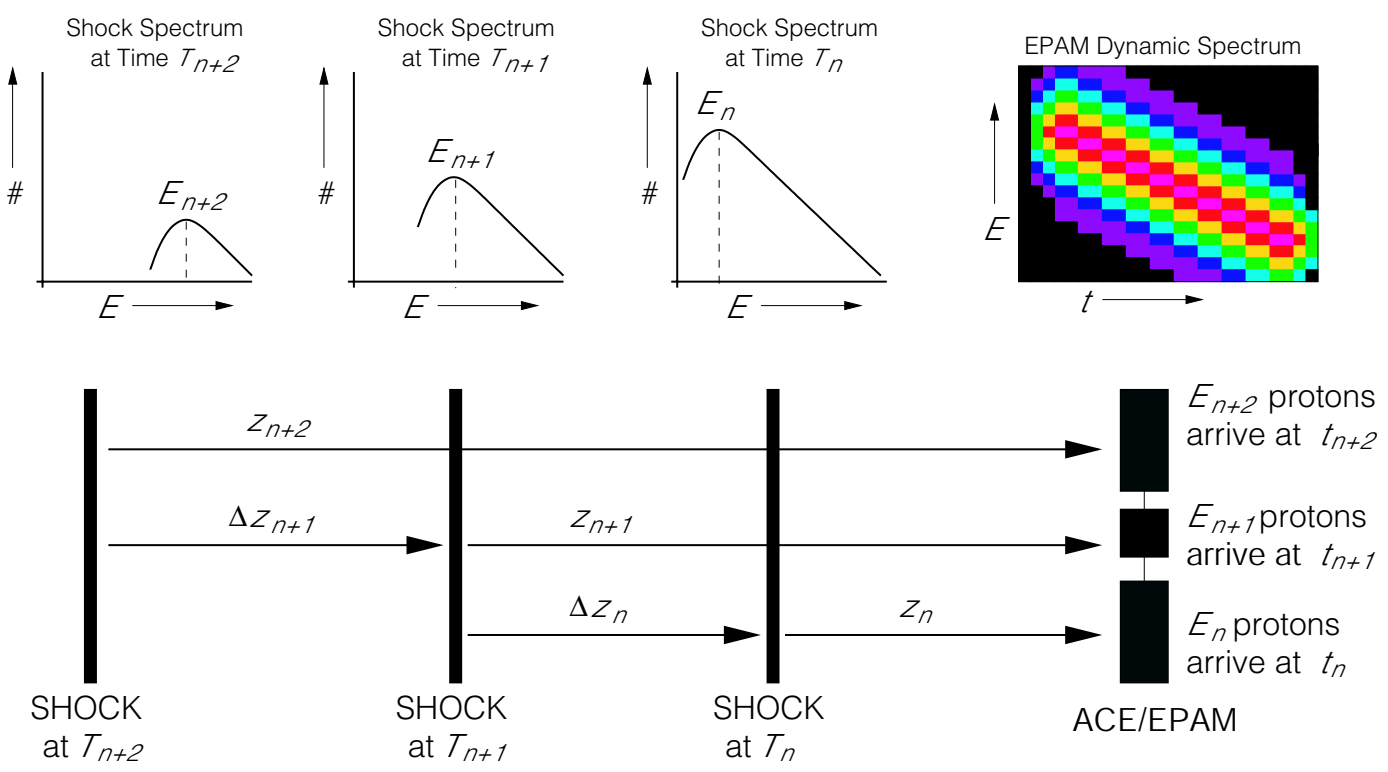

Fig. 4. The framework for our analysis and transform of the in-situ DSP into the shock's frame of reference. In this diagram we show a fraction of the shock's journey towards ACE where all distances $z_{n}$ and $\Delta z_{n}$ are measured along the interplanetary magnetic field. Although no spiral curvature is shown in this schematic, it is an implicit component of our framework.

This feature must be the result of proton acceleration by a CME-driven shock as it propagates through the inner heliosphere. The only other likely source for this proton population is the X-ray flare and would require that the evolution in the peak energy for the spectral peak was due to velocity dispersed propagation. This is hard to justify for the lower energy protons (e.g. $60-100 \mathrm{keV}$ ) which peak more than 2 days after the start of this event but exhibit highly field aligned pitch angle distributions. Such a delay requires these protons travelled an effective distance of 5-6 AU before reaching EPAM if they were released from the Sun at the time of the X-ray flare. This is at odds with the observed pitch angle anisotropy and we are left with the CME as the only remaining viable source of the DSP. With the DSP as the result of proton acceleration at a CME-driven shock, there is a good opportunity to study the effects of shock acceleration in isolation by focusing on this population alone. Here we simplify the DSP with the exponential line shown in black in Fig. 2d which was fitted by eye and provides a convenient way to describe the evolution of the peak energy for the shock accelerated proton population. Combining this information with in-situ observations of the shock's velocity we attempt to remove the effects of velocity dispersion, including the effects of scattering to learn how the peak proton energy accelerated by this CME-driven shock varies with the shock's position.

\section{Analysis}

\subsection{Theory}

Figure 4 describes the framework used for our analysis and the transform of the DSP into the shock frame of reference.
We adopt a piecewise approach where the exponential line fitted to the DSP (Fig. 2d) describes a set of proton energies $E_{n}$ which represent the peak in the proton energy spectrum at EPAM. DSP protons of energies $E_{n}$ were observed to arrive at EPAM at the set of times $t_{n}$ having travelled distances $z_{n}$ along the interplanetary magnetic field (IMF) and were originally released from the shock at times $T_{n}$. We denote the time at which the shock reaches EPAM as $t_{0}$ or equivalently $T_{0}$.

For any pair of points of the DSP we can construct a simple relation linking the difference in their arrival times to the distances they travelled, the speed of the shock $V_{z}(r)$ as measured along the IMF and the proton velocities $v_{n}$. The difference in EPAM arrival times $\Delta t=t_{n}-t_{n+1}$, can be expressed in terms of the proton and shock times of flight as

$\Delta t_{n, n+1}=\frac{z_{n}}{v_{n}}+\frac{\Delta z_{n}}{V_{z}(r)}-\frac{z_{n+1}}{v_{n+1}}$,

which, since $z_{n+1}=z_{n}+\Delta z_{n}$, can be re-written as

$\Delta t_{n, n+1}=\frac{z_{n}}{v_{n}}+\frac{\Delta z_{n}}{V_{z}(r)}-\frac{z_{n}+\Delta z_{n}}{v_{n+1}}$.

The value of $V_{z}(r)$ can be determined and extrapolated using in-situ solar wind speed measurements for the radial shock speed $V_{r}(r)$ whilst the same measurements provide an estimate of the IMF geometry which we use to convert between $V_{r}(r)$ and $V_{z}(r)$. Provided we know how $V_{r}(r)$ varies throughout the shock's journey to ACE, we can make this conversion for all $r$. For the purposes of this description we assume that $V_{r}(r)$ is a constant although any number of deceleration profiles might be employed. Using Eq. 2, starting at $n=0$ and using the proton energy from the spectral peak to set the velocities $v_{n}$ and $v_{n+1}$, we can derive a value for 
$z_{1}$. Incrementing $n$ and repeating this calculation allows us to gradually compile a list of values for $E_{n}$ as a function of $z_{n}$ and $T_{n}$ for all points of the DSP. Unfortunately, in using $E_{n}$ to calculate $v_{n}$ we are not making allowance for propagation effects; the competing actions of pitch angle scattering and focusing. To do so, we use Eq. 2 as a working basis and extend it to cover three consecutive points of the DSP instead of two.

Again, starting from the arrival of the shock at ACE and working backwards in time, we consider points $n=1,2$ and 3 from the DSP using

$\Delta t_{1,2}=\frac{z_{1}}{v_{1}}+\frac{\Delta z_{1}}{V_{z}(r)}-\frac{z_{1}+\Delta z_{1}}{v_{2}}$

and by letting $\Delta z_{2}=f_{1} \Delta z_{1}$ where $f_{1}$ is some constant we also have,

$\Delta t_{2,3}=\frac{z_{1}+\Delta z_{1}}{v_{2}}+\frac{f_{1} \Delta z_{1}}{V_{z}(r)}-\frac{z_{1}+\Delta z_{1}+f_{1} \Delta z_{1}}{v_{3}}$.

Physically, the constant $f_{n}$ is thus related to the evolution of the peak energy of the bump-on-the-tail protons released from the shock as it propagates. It is essentially an acknowledgement of the fact that for any given triplet of DSP points, the associated distances $\Delta z_{n}$ and $\Delta z_{n+1}$ are not necessarily equal. Inclusion of this constant $f_{n}$ is required such that we can use $\Delta z_{1} / V_{z}(r)$ as a common factor to couple these equations. Making $\Delta z_{1} / V_{z}(r)$ the subject for both Eqs. 3 and 4 then equating each to the other we have

$$
\begin{aligned}
\Delta t_{1,2} & -\frac{z_{1}}{v_{1}}+\frac{z_{1}+\Delta z_{1}}{v_{2}}= \\
\frac{1}{f_{1}} & {\left[\Delta t_{2,3}-\frac{z_{1}+\Delta z_{1}}{v_{2}}+\frac{z_{1}+\Delta z_{1}}{v_{3}}\right]+\frac{\Delta z_{1}}{v_{3}} . }
\end{aligned}
$$

Finally we re-arrange for $f_{1}$ to obtain

$$
\begin{aligned}
f_{1}= & {\left[\Delta t_{2,3}-\frac{z_{1}+\Delta z_{1}}{v_{2}}+\frac{z_{1}+\Delta z_{1}}{v_{3}}\right] } \\
& \times\left[\Delta t_{1,2}-\frac{z_{1}}{v_{1}}+\frac{z_{1}+\Delta z_{1}}{v_{2}}-\frac{\Delta z_{1}}{v_{3}}\right]^{-1},
\end{aligned}
$$

which forms the basis of our algorithm to include propagation effects during the protons' journey. Whilst the velocities $v_{1}, v_{2}$ and $v_{3}$ effectively remain unknown due to the effects of scattering, we take advantage of the fact that whilst the shock is close to EPAM, these effects must have been minimal. Consequently we take velocities $v_{1}$ and $v_{2}$ from the proton energies $E_{1}$ and $E_{2}$ and leave $v_{3}$ as a free parameter. All other parameters on the RHS of Eq. 6 can be obtained using Eq. 2 and we can calculate a value of $f_{1}$ for any value of $v_{3}$.

Crucially, the validity of any value of $f_{1}$ (and implicitly, any value for $v_{3}$ ), can be checked against the speed of the shock. We are in effect guessing a value for $v_{3}$, using this to calculate $f_{1}$ and then using $f_{1}$ to calculate a predicted shockspacecraft separation $z_{3}$ which can be checked against our model for the shock's propagation. The distance $z_{3}$ is given by $z_{1}+\Delta z_{1}+f \Delta z_{1}$, the time $T_{3}$ is given by $t_{3}-z_{3} / v_{3}$ also, we can transform the distance $z_{3}$ between the shock and ACE along the IMF, into a radial distance $r_{3}$ using a Parker spiral model. The difference between $r_{3}$ and the expected position of the shock at time $T_{3}$ constitutes an error in $v_{3}$. Minimizing this error must then give us a valid value for $v_{3}$ which takes propagation effects into account.

Having searched for and found valid values for $v_{3}, z_{3}$ and $T_{3}$, we are in a position to move on to the next triplet of points, $n=2,3$ and 4 and repeat the analysis. Continuing in this manner allows us to compile a list for $z_{n}$ and $T_{n}$ which does allow for the effects of scattering and focusing.

\subsection{Application}

Equation 6 was implemented numerically using the IDL programming language and for reasons of clarity, the procedure followed to map the DSP back into the shock reference frame is outlined qualitatively below.

1. Initially the bulk behaviour of the DSP must be quantified and was achieved by fitting an exponential to the DSP by eye. The small scale variations in the DSP are presumably due to small scale variations in the IMF between the shock and ACE. Since we rely upon the Parker model to describe the bulk configuration of the IMF, there seems no reason to quantify these relatively small variations and fitting the DSP in this way should provide suitable accuracy. Indeed, in practice, repeated attempts to fit the DSP do provide self-consistent results.

2. The velocity of the CME-driven shock in the spacecraft frame was determined as $422 \pm 31 \mathrm{~km} / \mathrm{s}$ at L1 by an incomplete fit (temperature information was not included) of the Rankine-Hugoniot relations (Qiang $\mathrm{Hu}$, private communication).

3. The initial values used in Eq. 6 i.e. for $n=1$ were calculated using Eq. 2 to determine values for: $z_{n}, \Delta z_{n}$ (and hence $z_{n+1}$ ), $T_{n}$ and $T_{n+1}$. To do so we use velocities $v_{n}$ and $v_{n+1}$ derived from the DSP proton kinetic energies $E_{n}$ and $E_{n+1}$. As such we are simultaneously solving the equations of motion for both the shock and DSP protons for $n=1$ and $n=2$.

4. A solution grid of values for $v_{n+2}$ was generated, together with a corresponding grid for $f_{n}$ calculated using Eq. 6 with the values determined above in step (3). The range of values in the solution grid can be reduced by realising that for small $\Delta t_{n, n+1}, v_{n+2}$ should not be greatly different from $v_{n+1}$, similarly, $f_{n}$ should not be less than or equal to zero and is highly unlikely to approach a larger value; we have used $f_{n}<50$ as a generous upper limit.

5. Each value for $f_{n}$ is used to predict the position of the shock which is compared with its position based upon the shock velocity calculated in step (2) at time $T_{n+2}$; 
the difference is used to represent the error in $v_{n+2}$. In practice, the solution grid was used to indicate approximately, the most suitable value for $v_{n+2}$, a Simplex algorithm was used to refine this value which was then used to determine values of $z_{n+2}$ and $T_{n+2}$. The distance $z_{n+2}$ is given by $z_{n+1}+f_{n} \Delta z_{n}$, whilst $T_{n+2}$ is given by $t_{n+2}-z_{n+2} / v_{n+2}$

Repeating from step (3) for the next three points of the DSP i.e. $n=2$, then again for $n=3$ and so on, for all points of the DSP, we derive a full set of values for the evolution of the spectral peak as a function of $z_{n}$ and $T_{n}$.

\section{Results}

Applying the procedure developed in Sect. 5 to the DSP yields the results shown in Fig. 5. In this instance we have used an upstream solar wind speed of $380 \mathrm{~km} / \mathrm{s}$ to constrain the Parker model for the IMF and a radial shock speed of $422 \mathrm{~km} / \mathrm{s}$. The DSP proton velocities $v_{1}$ and $v_{2}$ were derived from the kinetic energies $E_{1}$ and $E_{2}$. It is felt that this best represents the bulk characteristics of protons which contribute to the DSP around the time at which the shock reached L1. One might conceive of a scenario where proton velocities $v_{1}$ and $v_{2}$ require modification in order to reflect the bulk properties of the observed proton pitch angle distribution around the time the shock arrives at L1, which for example may have been peaked around a $45^{\circ}$ pitch angle. Although proton pitch angles might evolve between the shock and ACE for $n=1$ and $n=2$, this evolution should be insignificant for suitably small values of $\Delta t_{n, n+1}$. Nevertheless, during the 15-18 April event, the proton pitch angle distribution was anisotropic and largely anti-field-aligned whilst the shock was near ACE. As such we consider the initial velocities $v_{1}$ and $v_{2}$ do not require further modification by the pitch angle cosine.

The results shown in Fig. 5 were compiled using $\Delta t_{n, n+1}=1200 \mathrm{~s}$, equivalent to the MFSA time resolution and the time resolution of the normalised dynamic proton spectrum. Smaller values for $\Delta t_{n, n+1}$, down to $12 \mathrm{~s}$ do not provide any appreciable change to the results shown here and merely serve to increase the processing time. Figure 5 shows the transform results for 3 different deceleration profiles: constant shock velocity, constant deceleration and a deceleration which "switches off" at $\sim 0.68$ AU from the Sun which increases the shock speed near the Sun to about $750 \mathrm{~km} / \mathrm{s}$. The results for a transform which ignores the effects of scattering are also shown.

A pivotal aspect of the results of the CME-transform shown in Fig. 5 is the predicted time at which the highest energy $(\sim 1 \mathrm{MeV})$ DSP protons were released. In every scenario which includes the effects of scattering, the estimated time of release for these protons was before the 15 April CME erupted from the Sun. This is true for the constant shock velocity model and the situation does not improve by attempting a more realistic analysis which includes shock deceleration and implies significant propagational delays for
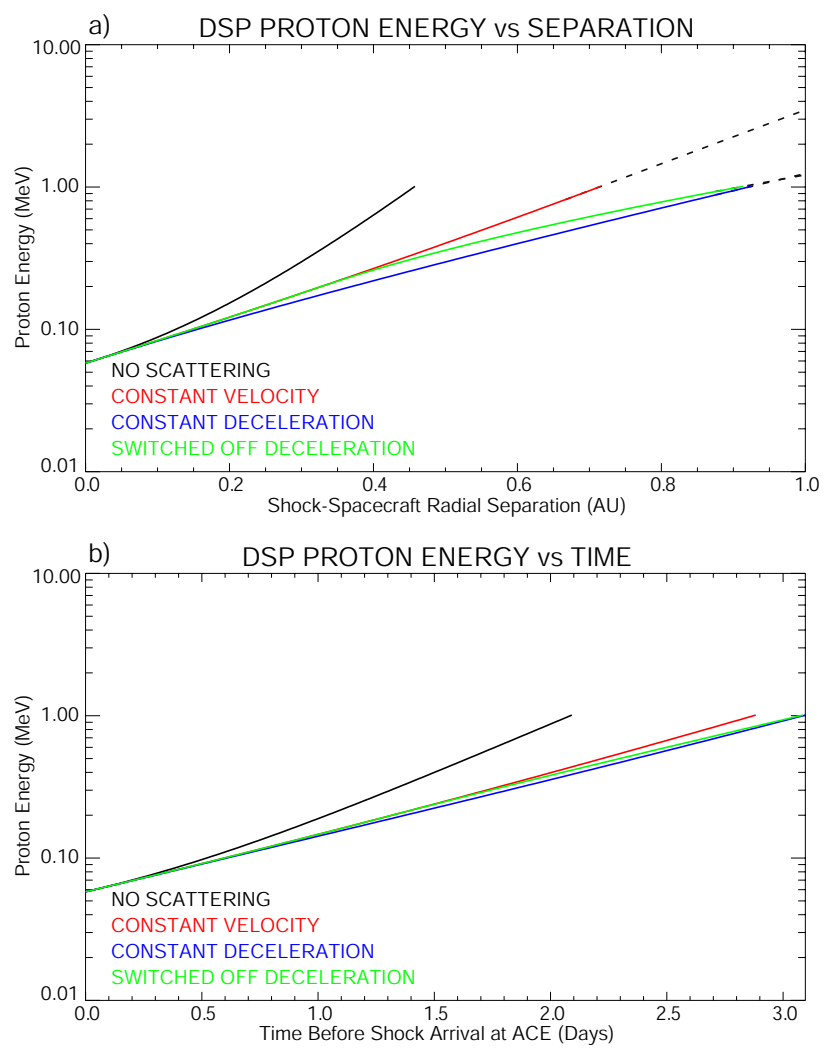

Fig. 5. Panel (a) shows, in the context of the bump-on-the tail, the most likely proton energy to be released from the midnight 18 April shock as a function of shock-ACE separation as measured along an ACE-Sun line. Panel (b) shows the most likely proton energy to be released from the shock as a function of time before the shock reached ACE. The black lines shows the results of the transform if we neglect the effects of scattering, all other solid lines include these effects for different models of the shock's propagation. The red line represents a constant shock velocity of $422 \mathrm{~km} / \mathrm{s}$, the blue line represents a constant deceleration from the Sun to L1 and the green line represents a constant deceleration out to $0.68 \mathrm{AU}$ from the Sun followed by constant velocity to L1.

these protons. If this was the case we must question the extent to which the 15 April CME contributed to the 15-18 April particle activity.

Figure 6 shows the effect of scattering and focusing on the DSP protons' apparent velocity which we have represented here as a mean pitch cosine. This representation has been used to describe the time of flight for the DSP protons compared to field aligned transport predictions. The algorithm provides information regarding where and when given DSP particles were released from the shock and it is a trivial matter to determine their actual time of flight compared to field aligned propagation. For example, a mean pitch angle cosine of 0.5 indicates the time of flight, having allowed for scattering effects for such a particle, is twice that expected for field aligned transport from the shock. Whilst this apparent velocity makes allowance for propagation effects, numerical modelling must be used 


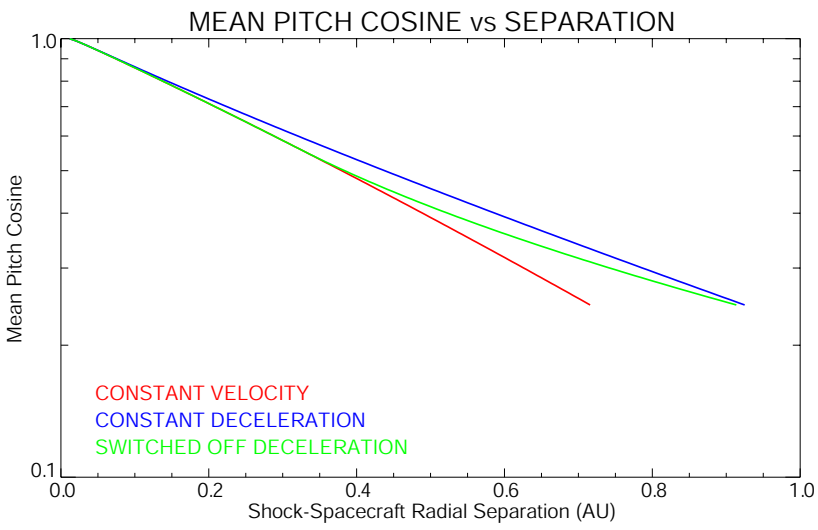

Fig. 6. Whilst the DSP protons might have field aligned velocities consistent with their energies, their apparent velocities as constrained by the propagation of the CME and their time of arrival at EPAM can be significantly different due to scattering. Here we consider the effective DSP proton velocities for $n>2$ i.e. the velocity prescribed by their time of flight over the shock-spacecraft separation along the IMF. These effective velocities are described here in terms of a mean pitch cosine. This can be considered a hypothetical pitch cosine for the protons that was maintained throughout their journey so as to be consistent with their time of flight from the shock to ACE. Again the coloured lines represent different models of shock propagation as indicated.

to determine more specific information regarding levels of interplanetary scattering; only the net delays introduced by scattering can be determined from our algorithm not the particle mean free paths. Figure 6 shows the mean pitch cosine against shock-spacecraft separation with the 3 different coloured lines corresponding to the 3 different models for the shock's propagation.

\section{Discussion}

Mathematically, the algorithm described in Sect. 5.2 appears to be limited only by the assumption that $V_{z}(r)$ does not change for 3 consecutive points of the DSP and the initial assumption that $v_{1}$ and $v_{2}$ are not altered by propagation effects. For a small enough value for $\Delta t_{n, n+1}$, these assumptions should not impair the validity of the analysis. Realistically, the analysis is limited to a greater extent by our lack of knowledge regarding changes in the shock's speed. In addition, the Parker spiral probably does not sufficiently describe the shock-spacecraft separation along the IMF throughout the event. This last factor may explain the slight kink in the DSP at around 00:00 UT 17 April 2001, alternatively this may be an inherent aspect of the shock's evolution, there appears to be no way to determine which is the more likely scenario from this event alone.

We have focused very closely on the DSP protons because they can be uniquely attributed to the associated CME-driven shock, nevertheless it is important to note that this bump-onthe-tail population should not by default, be considered the sole proton output from the shock. It seems likely this feature is simply an easily identifiable component of the particle population accelerated at MHD-shocks. Certainly its existence is predicted by the Rice et al. (2003) model for particle acceleration at CME-driven shocks. In modelling the escaping upstream spectra, a spectral peak is predicted in Rice et al. (2003) like that studied here which can be generated for strong and intermediate shocks and is seen over and above an underlying power-law population, also accelerated at the shock. They find the spectral peak is an enhancement which arises from protons escaping the shock after they have been trapped in the downstream flow. The evolution of their spectral peak is also predicted to evolve to lower energies with time. If the peak energy of the bump-on-the-tail scales with properties of the underlying power-law population, there is potential to gain further insight into the entire spectrum accelerated by the shock from the DSP observations.

The output from the Rice et al. (2003) model has been used for a Monte Carlo simulation of particle transport as described in $\mathrm{Li}$ et al. (2003) and provides a useful opportunity for quantitative comparison with the results of our analysis and EPAM's observations of the 15-18 April DSP at 1 AU. Two different characteristic shocks are modelled in $\mathrm{Li}$ et al. (2003): a "strong shock" and a "weak shock". The former shock moves at $1450 \mathrm{~km} / \mathrm{s}$ initially, and decelerates to $900 \mathrm{~km} / \mathrm{s}$ by the time it reaches $1 \mathrm{AU}$, the latter, starts at $900 \mathrm{~km} / \mathrm{s}$ dropping to $600 \mathrm{~km} / \mathrm{s}$ at $1 \mathrm{AU}$. As such the 15 April CME probably falls somewhere between the two.

Considering the observations at L1, the maximum energy of the DSP is $\sim 1-2 \mathrm{MeV}$ which was an order of magnitude less than the greatest peak energy of the bump-on-the-tail predicted by the Li et al. (2003) model for either strong or weak shocks. This DSP maximum was observed $8 \mathrm{~h}$ after the 15 April CME erupted. Taking this time of arrival into account we find more consistency with the Li et al. (2003) predictions but we must somehow explain the absence of higher energy DSP protons at L1. It is difficult to justify this absence considering the prompt arrival of relativistic protons following the 15 April activity.

The only evidence to suggest we may not have observed the more energetic DSP protons arises from the CMEtransform when used with a constant shock velocity profile which suggests we did not see DSP protons whilst the shock was more than $0.75 \mathrm{AU}$ from ACE. Nevertheless, if we extrapolate these results back to the Sun (i.e. we extrapolate the red line in Fig. 5a) we gain only a few MeV for the maximum DSP energy whereas we might hope to reach more than $10 \mathrm{MeV}$ in line with the Li et al. (2003) predictions. Furthermore, in attempting to make the transform more realistic by including only a moderate amount of shock deceleration, this extrapolated maximum starts to decrease and eventually approaches $\sim 1 \mathrm{MeV}$. By including significant deceleration to make the shock's journey more consistent with the required time of flight for the 15 April CME, the transform results predict an origin for the higher energy DSP protons which is either inside or behind the Sun. This appears to be true for all reasonable deceleration scenarios using the follow- 


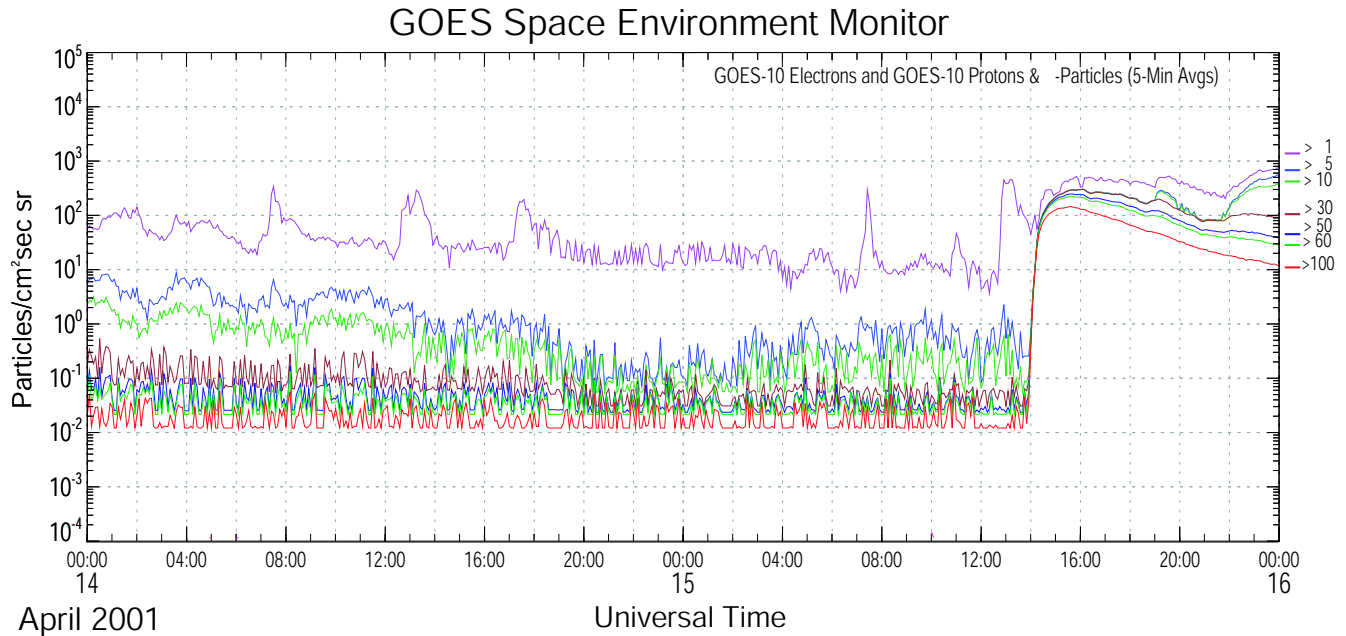

Fig. 7. This plot shows the GOES proton intensities for 14-15 April 2001 where the particle energies in MeV are given on the right (outside the plot panel), in $\mathrm{MeV}$. Energies above $5 \mathrm{MeV}$ are likely to be domionated by solar particles or those originating outside the heliosphere.

ing criteria: the shock must complete its journey to L1 after 2.43 days, the shock velocity at L1 must be $422 \mathrm{~km} / \mathrm{s}$ also the shock must possess a constant deceleration although that deceleration can "switch off" at some point inside L1 once it has reached $422 \mathrm{~km} / \mathrm{s}$. For this reason the possibility that an alternative CME drove the shock which arrived midnight 18 April has been considered.

The alternative CME must have left the Sun no later than $\sim 3$ days before midnight on the 18th to allow for the time at which $\sim 1-2 \mathrm{MeV}$ protons escaped the shock (using a constant shock velocity) as shown by the red line in Fig. 5b; introducing a CME deceleration seems to increase this upper time limit. Furthermore, it seems unlikely that the alternative $\mathrm{CME}$ could have erupted much earlier than midnight on the 14 April given the in-situ velocity measurements of this shock; with constant velocity, the time of flight for the shock observed at ACE at 00:04 18 April would have been 4.1 days. There were only 2 CME's observed by LASCO which fit these criteria and also possesed plane of sky speeds in excess of $400 \mathrm{~km} / \mathrm{s}$. One of these erupted around 20:53 UT 14 April from the east limb of the Sun and barely expands to reach the Earth-Sun line. As such it is unlikely that ACE was connected to this CME from the early stages of its journey and possibly, may never have observed its effects in-situ. This leaves the CME which erupted from the Sun's west limb at 17:23 UT April 14, presumably originating from the same region as the 15 April CME and with a plane of sky speed of $750 \mathrm{~km} / \mathrm{s}$.

The shock deceleration profiles in Fig. 5 have been tailored to match the observed properties of the 17:23 UT 14 April $\mathrm{CME}$ and do provide much more reasonable results. Again, the deceleration profiles used required a time of flight consistent with the time of the 14 April eruption and the shock arrival at midnight 18 April. One of the profiles uses constant deceleration throughout its journey to L1, the other stops decelerating at $\sim 0.68 \mathrm{AU}$ with a speed of $422 \mathrm{~km} / \mathrm{s}$ such that it can posses a velocity of $\sim 750 \mathrm{~km} / \mathrm{s}$ near the Sun in line with LASCO observations of the 14 April CME. With these models of shock velocity, the DSP can be traced back to $\sim 0.05$ AU from the Sun which is actually preferable to an origin which is closer to the Sun; the shock must have time to form and also, sufficient downstream turbulence must exist to delay the release of the DSP protons. In addition, if we attribute the 15-18 April DSP to the 14 April CME, the comparison with the Rice et al. (2003) model improves with respect to the properties of the bump-on-the-tail population. We also note that significant deceleration is required for the 15 April CME-driven shock to reach L1 with a speed of $422 \mathrm{~km} / \mathrm{s}$ given a required, average velocity of $700 \mathrm{~km} / \mathrm{s}$, necessary to satisfy the time of flight to reach L1 at midnight 18 April. This is in contrast to the Tappin et al. (2004) case study focusing on a May 2003 CME that was tracked using the Solar Mass Ejection Imager (SMEI); an "all sky" coronagraph. The results of this case study suggested the CME in question propagated from the Sun to $1 \mathrm{AU}$ with an almost constant velocity of $\sim 900 \mathrm{~km} / \mathrm{s}$. This would appear to support a scenario where the 14 April CME preceeds the 15 April CME and presumably retards it significantly. The linear extrapolation of the two CME height-time profiles (Fig. 3) also supports this scenario provided they both travel within similar regions of the heliosphere. There is, however, some discrepancy regarding the underlying power-law population suggested by the Rice et al. (2003) model for a shock driven by a CME such as that on 14 April. The maximum energetic extent of such a power law is expected to reach energies of $\sim 100 \mathrm{MeV}$ although there is no evidence of such protons in the GOES dataset until the onset of the 15 April activity, as shown in Fig. 7. We also note that the results of the CME-transform do require significant propagational delays for the higher energy DSP protons at some point in their journey from the shock. 


\section{Conclusion}

The analysis and results presented here may provide particularly useful constraints when combined with models of particle acceleration precisely because we have attempted to transform in-situ observations into the shock frame of reference. Given that the DSP is expected to be a defining characteristic for strong and also some weaker shocks within the Rice et al. (2003) framework, transformation of the DSP into the shock frame provides a way to investigate models of particle acceleration whilst removing the effects of particle transport. Models could be more accurately evaluated against such analysis throughout their journey rather than merely at a given in-situ location. For the same reason, this transformation should help constrain models of particle transport by specifying the time and place at which the DSP protons were able to escape the shock directly from observations providing a rigorous set of boundary conditions for the protons' journey, if we can determine how the shock velocity changes.

We find good qualitative agreement with the theoretical description of these events provided by Rice et al. (2003) and $\mathrm{Li}$ et al. (2003). In general there is quantitative agreement for the observed DSP properties and their predicted properties, although this does depend somewhat upon which shock we choose as the driver for the 15-18 April DSP. For the 15 April CME we find agreement only if we failed to observe a higher energy component of the DSP and this seems unlikely given a good magnetic connection to the Sun. The best agreement arises if we attribute the DSP to the 14 April CME which, encouragingly, is also consistent with the output of the CMEtransform presented here. For all realistic teatments of the 15 April CME propagation, the results of the transform become non-physical for the higher energy component of the DSP. In comparison, the results for the 14 April CME are a considerable improvement. The highest energy of the DSP is mapped back to $\sim 0.05 \mathrm{AU}$ from the Sun which allows time for the shock and downstream turbulence to form. Also, a straightforward shock deceleration profile can be employed to reach appropriate CME speeds near the Sun, without producing any non-physical results. There are also some discrepancies, for example, we do not observe the $\sim 10-100 \mathrm{MeV}$ protons from the underlying power-law that are expected to accompany the DSP generated by the 14 April CME-driven shock within the Rice et al. (2003) model. Also, we find the results of the transform produce propagation delays for the higher energy DSP protons that are larger than had been expected; of course this is an inescapable consequence of linking the DSP to the 14 April CME.

Thus the application of the CME-transform in this case raises some interesting possibilities. Counter-intuitively it suggests the 15 April CME, and associated flare did not generate all of the particle activity at L1 during 15-18 April. We have focused on the 14 April CME as the source of the DSP as it appears to be the most likely alternative based upon the LASCO observations but it is worth considering that other relevant ejections may have been lost amongst the variety of activity around that time. There may also have contempo- rary magnetic ejections which accompanied this activity but were too faint to be observed by LASCO, as considered in Lyons and Simnett (2001). Despite this, the results of our analysis and a favourable comparison with models that predict the presence of a DSP, support a scenario where the bulk of the particle output was either generated or at least modulated by an alternative CME. It is difficult to see how the transform itself might be at fault in these predictions as the principal assumptions involved do not appear to be unreasonable or flawed although we cannot be so certain about the framework we have used. A level of interplay between the output of the shock which reached L1 at midnight 18 April and the 15 April CME is possible and has not been allowed for within the framework of the transform. Indeed it is difficult to know how one might include these effects if, for example, the output of the 15 April activity augmented the output from the shock which reached L1 at midnight 18 April. Clearly it would be beneficial to investigate the results of the CME transform for a number of "purer" DSP events but this is beyond the scope of this study. Doubtless the analysis of such DSP signatures would also benefit from improved coverage of CME propagation, either from SMEI or future heliospheric imagers.

Acknowledgements. Both authors would like to acknowledge the work carried out by the EPAM instrument team and in particular the useful software, perspectives and advice offered by S. J Tappin. I. M. R. would like to thank C. Hooley for his advice during the formative stages of our analysis and also the referees for their comments which prompted such an interesting interpretation.

Topical Editor R. Forsyth thanks K. Rice and another referee for their help in evaluating this paper.

\section{References}

Bieber, J. W., Evenson, P., Dröge, W., Pyle, R., Ruffolo, D., Rujiwarodom, M., Tooprakai, P., and Khumlumlert, T.: Spaceship earth observations of the Easter 2001 solar particle event, ApJ, 601, L103-L106, 2004.

Brueckner, G. E., Howard, R. A., Koomen, M. J., Korendyke, C. M., Michels, D. J., Moses, J. D., Socker, D. G., Dere, K. P., Lamy, P. L., Llebaria, A., Bout, M. V., Schwenn, R., Simnett, G. M., Bedford, D. K., and Eyles, C. J.: The Large Angle Spectroscopic Coronagraph (LASCO), Sol. Phys., 162, 357-402, 1995.

Gold, R. E., Krimigis, S. M., Hawkins III, S. E., Haggerty, D. K., Lohr, D. A., Fiore, E., Armstrong, T. P., Holland, G., and Lanzerotti, L. J.: Electron Proton and Alpha Monitor on the Advanced Composition Explorer, Space Sci. Rev., 86, 541-562, 1998.

Klein, K.-L. and Trottet, G.: The origin of solar energetic particle events: coronal acceleration versus shock wave acceleration, Space Sci. Rev., 95, 215-225, 2001.

Li, G., Zank, G. P., and Rice, W. K. M.: Energetic particle acceleration and transport at coronal mass ejection-driven shocks, J. Geophys. Res., 108, 2003.

Lyons, M. A. and Simnett, G. M.: Erupting magnetic structures observed with SoHO/LASCO, Sol. Phys., 200, 203-211, 2001.

McComas, D. J., Bame, S. J., Barker, P., Feldman, W. C. Phillips, J. L., Riley, P., and Griffee, J. W.: Solar wind electron proton 
and alpha monitor (SWEPAM) for the Advanced Composition Explorer, Space Sci. Rev., 86, 563-612, 1998.

Reames, D. V.: Particle acceleration at the Sun and in the heliosphere, Space Sci. Rev., 90, 413-491, 1999.

Rice, W. K. M., Zank, G. P., and Li, G.: Particle acceleration and coronal mass ejection driven shocks: Shocks of arbitrary strength, J. Geophys. Res., 108, 2003.

Simnett, G. M.: A new concept for solar flares, in ESA SP-535: Solar Variability as an Input to the Earth's Environment, 613618, 2003.

Smith, C. W., L'Heureux, J., Ness, F., N., Acuna, M. H., Burlaga, L. F., Scheifele, and J.: The ACE magnetic fields experiment, Space Sci. Rev., 86, 613-632, 1998.
Tappin, S. J., Buffington, A., Cooke, M. P., Eyles, C. J., Hick, P. P., Holladay, P. E., Jackson, B. V., Johnston, J. C., Kuchar, T., Mizuno, D., Mozer, J. B., Price, S., Radick, R. R., Simnett, G. M., Sinclair, D., Waltham, N. R., and Webb, D. F.: Tracking a major interplanetary disturbance with SMEI, Geophys. Res. Let., 31, 2802-2806, 2004.

Torsti, J., Kocharov, L., Laivola, J., Lehtinen, N., Kaiser, M. L., and Reiner, M. J.: Solar particle event with exceptionally high ${ }^{3} \mathrm{He}$ enhancement in the energy range up to $50 \mathrm{MeV} /$ nucleon, ApJ, 573, L59-L63, 2002.

Tylka, A. J., Boberg, P. R., Cohen, C. M. S., Dietrich, W. F., Maclennan, C. G., Mason, G. M., Ng, C. K., and Reames, D. V.: Flare- and shock-accelerated energetic particles in the solar events of 2001 April 14 and 15, ApJ, 581, L119-L123, 2002. 